Space Ops 2014, Pasadena, California

Abstract

\title{
Asteroid Redirect Crewed Mission Nominal Design and Performance
}

\author{
Gerald L. Condon ${ }^{1}$, Jacob Williams ${ }^{2}$ \\ ${ }^{1}$ NASA Johnson Space Center, Houston, TX 77058 \\ ${ }^{2}$ ERC, Inc., JETS Contract Group, Houston, TX 77058
}

In 2010, the President announced that, in 2025, the U.S. intended to launch a human mission to an asteroid [1]. This announcement was followed by the idea of a Capability Driven Framework (CDF) [2], which is based on the idea of evolving capabilities from less demanding to more demanding missions to multiple possible destinations and with increased flexibility, cost effectiveness and sustainability. Focused missions, such as a NASA inter-Center study that examined the viability and implications of sending a crew to a Near Earth Asteroid (NEA) [3], provided a way to better understand and evaluate the utility of these CDF capabilities when applied to an actual mission. The long duration of the NEA missions were contrasted with a concept described in a study prepared for the Keck Institute of Space Studies (KISS) [4] where a robotic spacecraft would redirect an asteroid to the Earth-Moon vicinity, where a relatively short duration crewed mission could be conducted to the captured asteroid. This mission concept was included in the National Aeronautics and Space Administration (NASA) fiscal year 2014 budget request, as submitted by the NASA Administrator [5]. NASA studies continued to examine the idea of a crewed mission to a captured asteroid in the Earth-Moon vicinity. During this time was an announcement of NASA's Asteroid Grand Challenge [6]. Key goals for the Asteroid Grand Challenge are to locate, redirect, and explore an asteroid, as well as find and plan for asteroid threats. An Asteroid Redirect Mission (ARM) study was being conducted, which supports this Grand Challenge by providing understanding in how to execute an asteroid rendezvous, capture it, and redirect it to Earth-Moon space, and, in particular, to a distant retrograde orbit (DRO). Subsequent to the returning of the asteroid to a DRO, would be the launch of a crewed mission to rendezvous with the redirected asteroid. This report examines that crewed mission by assessing the Asteroid Redirect Crewed Mission (ARCM) nominal design and performance costs associated with an Orion based crewed rendezvous mission to a captured asteroid in an Earth-Moon DRO.

The ARM study includes two fundamental mission phases: 1) The Asteroid Redirect Robotic Mission (ARRM) and 2) the ARCM. The ARRM includes a solar electric propulsion based robotic asteroid return vehicle (ARV) sent to rendezvous with a selected near Earth asteroid, capture it, and return it to a DRO in the Earth-Moon vicinity. The DRO is selected over other possible asteroid parking orbits due to its achievability (by both the robotic and crewed vehicles) and by its stability (e.g., no orbit maintenance is required). After the return of the asteroid to the Earth-Moon vicinity, the ARCM is executed and carries a crew of two astronauts to a DRO to rendezvous with the awaiting ARV with the asteroid. The outbound and inbound transfers employ lunar gravity assist (LGA) flybys to reduce the Orion propellant requirement for the overall nominal mission, which provides a nominal mission with some reserve propellant for possible abort situations.

The nominal mission described in this report provides a better understanding of the mission considerations as well as the feasibility of such a crewed mission, particularly with regard to spacecraft currently undergoing development, such as the Orion vehicle and the Space Launch System (SLS). 
[1] Obama, B.H., "Remarks by the President on Space Exploration in the 21st Century, "John F. Kennedy Space Center, April 15, 2010, (http://www.whitehouse.gov/the-press-office/remarkspresident-space-exploration-21st-century).

[2] "Human Space Exploration Framework Summary”, National Aeronautics and Space Administration, January 12, 2011,

[3] Drake, B., "Strategic Implications of Human Exploration of Near-Earth Asteroids", IEEAC Paper 1069; JSC-CN-25037, IEEE 2012 Aerospace Conference, Big Sky, MT, 3-10 Mar. 2012, United States.

[4] Brophy, J., Culick, F., Friedman, L., et al., \Asteroid Retrieval Feasibility Study," Technical Report, Keck Institute for Space Studies, California Institute of Technology, Jet Propulsion Laboratory, April 2012.

[5] Ramsey, S., Kraft, R., “NASA Announces Asteroid Grand Challenge”, Release: 13-188, NASA HQ, June 18, 2013. http://www.nasa.gov/home/hqnews/2013/jun/HQ_13188_Asteroid_Grand_Challenge.html

[6] 6/18/13: NASA Announces Asteroid Grand Challenge Release: 12-188, NASA HQ 


\section{Chronology}

1) 4/15/10: President announces U.S. (et. al.?) will send first crew to an asteroid. At Kennedy Space Center

a. http://www.nasa.gov/about/obamaspeechfeature.html

b. "And by 2025, we expect new spacecraft designed for long journeys to allow us to begin the first-ever crewed missions beyond the moon into deep space. We'll start by sending astronauts to an asteroid for the first time in history.”

c. Crew goes to asteroid.

d. Obama, B.H., "Remarks by the President on Space Exploration in the 21st Century, "John F. Kennedy Space Center, April 15, 2010, (http://www.whitehouse.gov/the-pressoffice/remarks-president-space-exploration-21st-century).

2) 1/12/11: Capability Driven Framework:

a. "Human Space Exploration Framework Summary", National Aeronautics and Space Administration, January 12, 2011, http://www.nasa.gov/exploration/new_space_enterprise/home/heft_summary.html

3) March 3-10, 2012: Drake, B., "Strategic Implications of Human Exploration of NearEarth Asteroids”, IEEAC Paper 1069; JSC-CN-25037, IEEE 2012 Aerospace Conference, Big Sky, MT, 3-10 Mar. 2012, United States.

4) April 2012: Keck Study of feasibility of asteroid capture and return April 2012

a. Brophy, J., Culick, F., Friedman, L., et al., \Asteroid Retrieval Feasibility Study," Technical Report, Keck Institute for Space Studies, California Institute of Technology, Jet Propulsion Laboratory, April 2012.

b. Capture asteroid, bring back, crew to Earth vicinity for asteroid visit

5) Date??: ARM - JPL

6) January 2013 - August 2013: CAT study, NASA JSC/JPL/GRC/LaRC

7) 4/10/13: NASA Administrator Bolden's Statement on the NSA FY 2014 Budget Request, Release: 13-104, NASA HQ

a. http://www.nasa.gov/home/hqnews/2013/apr/HQ_13104_Bolden_FY14_Budget_Statement.html

8) 6/18/13: NASA Announces Asteroid Grand Challenge Release: 12-188, NASA HQ 